\title{
Seven novel and six de novo $P H E X$ gene mutations in patients with hypophosphatemic rickets
}

\author{
SHAN-SHAN LI ${ }^{1,2}$, JIE-MEI GU ${ }^{1,2}$, WEI-JIA YU ${ }^{1,2}$, JIN-WEI HE ${ }^{1,2}$, WEN-ZHEN FU ${ }^{1,2}$ and ZHEN-LIN ZHANG ${ }^{1,2}$ \\ ${ }^{1}$ Metabolic Bone Disease and Genetics Research Unit, Department of Osteoporosis and Bone Diseases, \\ Shanghai Jiao Tong University Affiliated Sixth People's Hospital; ${ }^{2}$ Shanghai Key Clinical Center \\ for Metabolic Disease, Shanghai 200233, P.R. China
}

Received August 23, 2015; Accepted October 18, 2016

DOI: $10.3892 /$ ijmm.2016.2796

\begin{abstract}
Inactivating mutations in phosphate-regulating gene with homologies to endopeptidase on the $\mathrm{X}$ chromosome (PHEX) have been identified as a cause of X-linked hypophosphatemic rickets (XLH; OMIM 307800). In the present study, we enrolled 43 patients from 18 unrelated families clinically diagnosed with hypophosphatemic rickets and 250 healthy controls. For each available individual, all 22 exons with their exon-intron boundaries of the PHEX gene were directly sequenced. The levels of serum fibroblast growth factor 23 (FGF23) were measured as well. Sequencing analysis detected 17 different $P H E X$ gene mutations, and 7 of these were identified as novel: 3 missense mutations, including c.304G >A (p.Gly102Arg) in exon 3, c.229T >C (p.Cys77Arg) in exon 3 and c.824T $>C$ (p.Leu275Pro) in exon 7; 2 deletion mutations, including c.528delT (p.Glu177LysfsX44) in exon 5 and c.1234delA (p.Ser412ValfsX12) in exon 11; and 2 alternative splicing mutations, including c.436_436+1delAG in intron 4 at splicing donor sites and c.1483-1G $>\mathrm{C}$ in intron 13 at splicing acceptor sites. Moreover, 6 mutations were proven to be de novo in 6 sporadic cases and the probands were all females. No mutations were found in the 250 healthy controls. The serum levels of FGF23 varied widely among the patients with $\mathrm{XLH}$, and no significant difference was found when compared with those of the healthy controls. On the whole, the findings of this study provide new insight into the spectrum of PHEX mutations and provide potential evidence of a critical domain in PHEX protein. In addition, the finding of an overlap of the
\end{abstract}

Correspondence to: Professor Zhen-Lin Zhang, Metabolic Bone Disease and Genetics Research Unit, Department of Osteoporosis and Bone Diseases, Shanghai Jiao Tong University Affiliated Sixth People's Hospital, 600 Yishan Road, Shanghai 200233, P.R. China E-mail:zz12002@medmail.com.cn

Key words: X-linked hypophosphatemic rickets, phosphate-regulating gene with homologies to endopeptidase on the $\mathrm{X}$ chromosome, mutation, fibroblast growth factor 23 serum FGF23 levels between the patients with XLH and the healthy controls indicates its limited diagnostic value in XLH.

\section{Introduction}

Inherited hypophosphatemic rickets, characterized by bone mineralization disorders due to hypophosphatemia, secondary to a leak of phosphate from the kidneys, was first introduced by Albright et al (1). The clinical characteristics of the disease include growth retardation, bending of the weight-bearing extremities, and the resistance to vitamin D therapy during early childhood. Subsequently, patients suffer from spontaneous dental abscesses, extensive bone pain and arthropathy with aging. X-linked hypophosphatemic rickets (XLH; OMIM 307800), with an occurrence of appproximately 1 in 20,000 live births, is known as the most prevalent form of inherited hypophosphatemic rickets $(2,3)$. The mutation in the phosphate-regulating gene with homologies to endopeptidase on the X chromosome (PHEX; MIM 300550) was identified in 1995 as the causative gene for the disease (4). Thereafter, the genetic basis of other less prevalent types of inherited hypophosphatemic rickets, such as autosomal dominant hypophosphatemic rickets (ADHR; OMIM 193100) and autosomal recessive hypophosphatemic rickets (ARHR1; OMIM 241520), was successively clarified $(5,6)$. Nevertheless, the underlying pathogenesis for some sporadic cases without known genetic mutations remains to be determined and the significant findings of key roles of microRNAs (miRNAs of miRs) involved in bone formation may provide potential research issues (7-9).

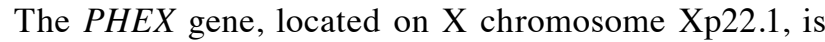
composed of 22 exons spanning $243 \mathrm{~kb}$ and encodes for 749 amino acid proteins (10). It exhibits great homology to the M13 zinc metallopeptidases, a class of type 2 integral membrane glycoproteins that includes neprilysin $(N E P)$, endothelin-converting enzymes 1 and 2 (ECE-1 and ECE-2), as well as the Kell antigen (KELL) (11). These proteins show the common structural traits of a short N-terminal cytoplasmic domain, a single transmembrane hydrophobic region and a large extracellular domain. The latter contains 10 highly conserved cysteine residues and two zinc-binding motifs and is involved in the secondary structure conformation or catalytic activity of the protein (11). It has been demonstrated that the tissuespecific expression of the $P H E X$ gene and its mRNA expression 
is predominantly detected in bone lineage cells; for instance, osteoblasts, osteocytes and odontoblasts $(10,12)$. However, the exact mechanisms through which inactivated $P H E X$ leads to abnormal skeletal and renal manifestations in XLH have not yet been fully clarified. Studies have suggested the existence of phosphatonin that is responsible for the regulation of phosphate homeostasis in the Hyp mouse (13), an animal model of XLH with a 3 ' deletion of the $P H E X$ gene. Based on the finding of increased fibroblast growth factor 23 (FGF23) transcripts in the Hyp mouse, FGF23 was then regarded as a leading candidate for phosphatonin downstream of PHEX (14).

An extensive mutation analysis of the genetic defects of the PHEX gene only revealed a few cases in China (15-21). In the present study, we screened a total of 18 affected families for mutations in the PHEX gene in order to interpret the mutation traits in Chinese patients and potentially provide evidence of a critical domain in PHEX protein. Moreover, the serum FGF23 levels in affected individuals were also measured as this may also contribute to our understanding of the molecular basis of XLH.

\section{Materials and methods}

Ethics statement and study subjects. This study was approved by the Ethics Committee of the Shanghai Jiao Tong University Affiliated Sixth People's Hospital (Shanghai, China) and all subjects or their guardians (for the under-aged participants) provided written informed consent prior to enrollment. The subjects enrolled in this study were from the Department of Osteoporosis and Bone Diseases in Shanghai Jiao Tong University Affiliated Sixth People's Hospital, Shanghai, China over the past 3 years. A total of 18 unrelated Chinese families with hypophosphatemic rickets was examined and all subjects were of Han ethnicity. The pedigrees of the families with hypophosphatemic rickets are shown in Fig. 1. The diagnosis of hypophosphatemic rickets was mainly based on a history of childhood rickets, clinical manifestations and biochemical tests indicating hypophosphatemia along with elevated levels of serum alkaline phosphatases. Patients with secondary rickets due to malnutrition, medication or tumor-induced osteomalacia were excluded. Finally, 65 individuals, including 43 patients, and 250 unrelated healthy controls were recruited and subjected to blood sampling followed by DNA analyses. In addition, another 95 healthy controls with normal serum phosphate and calcium levels and normal renal function were randomly selected to carry out measurements of serum levels of FGF23 in order to derive a reference range.

Mutation screening. To detect mutations in the PHEX gene, all DNA samples from the probands were initially analyzed. When a $P H E X$ mutation was confirmed, DNA from first-degree relatives, as well as the symptomatic individuals was then screened for the detected mutation. A proband was identified as a sporadic case if the PHEX mutation was not detected in the parents, namely if both were asymptomatic.

Mutation analyses of the affected individuals were performed by the direct sequencing of polymerase chain reaction (PCR) products amplified from genomic DNA. The DNA was extracted from peripheral white blood cells by proteinase $\mathrm{K}$ digestion followed by purification with phenol/chloroform and isopropyl alcohol precipitation. The DNA sequence for the $P H E X$ gene was obtained from the available online database (NCBI Reference Sequence Accession no. NG_007563.2). All the 22 exons with their adjacent intronic sequences of the PHEX gene were amplified by PCR with 21 pairs of sequencing primers designed using Primer 3 software (http://bioinfo.ut.ee/ primer3-0.4.0/). The primer sequences are presented in Table I. Direct sequencing was performed using the BigDye Terminator Cycle Sequencing Ready Reaction kit, version 3.1 and the PCR products were directly sequenced using an automated ABI PRISM 3130 sequencer (both from Applied Biosystems, Foster City, CA, USA). Simultaneously, single-nucleotide polymorphisms (SNPs) were identified using PolyPhred (http:// droog.gs.washington.edu/polyphred/) and novel mutations were identified using HGMD (http://www.hgmd.cf.ac.uk/). Mutations were checked using Mutalyzer 2.0 (http://mutalyzer.nl/check). The DNA sequences obtained were aligned with homologous sequences that had been deposited into GenBank using the CluxtalX 1.83 algorithm. To predict the impact of missense mutations on protein structure and function, Polymorphism Phenotyping v2 (PolyPhen-2; http://genetics.bwh.harvard.edu/ pph2) (22) and Sorting Tolerant from Intolerant (SIFT; http:// sift.jcvi.org) (23) were used based on sequence alignments.

Measurement of circulating intact FGF23 levels. Serum FGF23 leels were measured using an intact human FGF23 enzyme-linked immunosorbent assay (ELISA) kit obtained from Kainos Laboratories Inc. (Tokyo, Japan). The detectable concentration ranged from 3 to $800 \mathrm{pg} / \mathrm{ml}$. This assay has been validated in previous studies $(16,24)$.

Statistical analyses. Normally distributed variables are presented as the means $\pm \mathrm{SD}$, and non-normally distributed variables as the median (2.5th and 97.5th percentiles). Comparisons between groups were made using unpaired Student's t-tests for normal data, and the Wilcoxon rank sum test for non-normal data. Values of $\mathrm{P}<0.05$ were considered to indicate statistically significant differences. Statistical analyses were performed using SPSS 13.0 software.

\section{Results}

The baseline clinical data and PHEX gene mutation analyses of 65 participants originating from 18 families are summarized in Table II. A total of 43 patients was confirmed to have XLH according to their clinical and genetic evidence. All the patients exhibited varying degrees of growth retardation, bending legs, dental anomalies, hypophosphatemia, markedly elevated serum alkaline phosphatase levels and normal serum calcium. The median (25th and 75th percentiles) age of the patients was $22.0(8.3,29.5)$ years and the median serum phosphate levels were $0.70(0.57,0.80) \mathrm{mmol} / \mathrm{l}$.

We identified 17 different mutations in the PHEX gene from 18 unrelated families and the distribution of $P H E X$ mutations were 6 alternative splicing mutations (35.3\%), 5 missense mutations (29.4\%), 3 nonsense mutations (17.6\%), 2 deletion mutations (11.8\%) and 1 insertion mutation (5.9\%) (Fig. 2). Among these, 7 mutation sites were identified as novel and they were respectively detected from families $4,6,7,8,12$, 13 and 16: c.1483-1G>C in intron 13 at splicing acceptor sites 
Table I. List of primer sequences used for PCR amplification of the PHEX gene.

\begin{tabular}{rll}
\hline Exons & \multicolumn{1}{c}{ Forward primer $\left(5^{\prime} \rightarrow 3^{\prime}\right)$} & \multicolumn{1}{c}{ Reverse primer $\left(5^{\prime} \rightarrow 3^{\prime}\right)$} \\
\hline 1 & AGGGACTTTGCTGAGGGAGAG & CCACTCGAAGCCACTTACACC \\
2 & TGGGTTTTGGAATACCGTGTC & AAGAGAGGCCATTCAGCCTTC \\
3 & CAAGGCTTGGAAACTGGTTGA & TTATGTTGAGATCTGGGAGTCCA \\
4 & GGCACCATATGTGGGTGGATA & GTTTGCCCTGCTGACTTTGTC \\
5 & CACATTGAAGCGTGGATCGTA & CGGGAGAAGGGAATATTCTGG \\
6 & GCTCTGCCCAATCATGTTACC & GCAGCCTGGTAAGGCACATAG \\
7 & GGGTGCCTGGTATTGCATAAT & CCAATGGGCAATGACACAAA \\
8 & ACCACACCAAAGCCTTGAAAA & GAGCCAATGCCAACAATTACC \\
9 & GGATGGCAATGATCAGGAGTT & GACAGTGCTTTTGGCAGTTC \\
10 & ATGTTCACTCTGAGGGCTGGA & GGCTACAAACTCCCCCTGTCT \\
11 & CAGCCATGGGTTTTATCCAAA & CCCACTCCCCTGGAAAACTAC \\
12 & AGTGTTGCCAGAGCATGGAGT & AGGAAAGGCCGAATTACAAGG \\
13 & TCGATTCAGTCACCTTCTCCA & GAAAGGCACAAGGCCAGTAAA \\
14 & TGACTGATGCAGCTTCTCTGC & ATGCTAGAAATGGGGGACCTG \\
15 & GCAGGGACAGCCCTTTAGATT & GCCACTTTTGGGGGAAATAAG \\
16 & GTGCAAAATGGTTTCCTGAA & GTCCAGCCATACACCCTGGTA \\
17 & AAGCAGTTTATCTTGGCTTTCCA & CAAGCCATCACAGCAAGACAC \\
18 & CTGCTTTTTGAAGGCTTGTCG & ATGCCTGGTTAAGGGATGACC \\
19 & TTGATGCCTCTTGCTGAATGA & AAATGAACCTAGCCCCAAGGA \\
20 & TGGTAAGCAACAGGACATGGA & AGGGCTGCTAACCCATTTGAT \\
21 & TTCCTGGGCACATATACGATTC & TTTTGGCTGCAAAATGGAAAT \\
22 & CAGAACCTGTTGATGTGCAAGA & GCCAACACCCTAAAATGGACA \\
\hline
\end{tabular}

PCR, polymerase chain reaction; PHEX, phosphate-regulating gene with homology to endopeptidase on the X chromosome.

leading to truncated protein, c.1234delA (p.Ser412ValfsX12) in exon 11, c.436_436+1delAG in intron 4 at splicing donor sites leading to truncated protein, c.824T $>\mathrm{C}$ (p.Leu275Pro) in exon 7 , c.304G $>$ A (p.Gly102Arg) in exon 3, c.229T $>C$ (p.Cys77Arg) in exon 3 and c.528delT (p.Glu177LysfsX44) in exon 5. PolyPhen-2 and SIFT were performed to assess the missense mutational consequence of $P H E X$, and all 3 missense mutations were predicted to be probably damaging with a score of 0.930 for L275R, 0.917 for G102R and 1.000 for C77R, and a SIFT score of $<0.05$. Notably, the amino acid residues at p.275, p.102 and p.77 were evolutionarily highly conserved across 12 different species as shown in Fig. 3. In addition, the probands from families $9,10,11,16,17$ and 18 were identified as sporadic cases and the detected PHEX mutations were likely to be de novo, and the 6 probands were all female patients.

Serum FGF23 levels were not normally distributed in either the normal controls or the XLH patients. The median value for intact serum FGF23 levels from the 95 healthy individuals aged between 22 to 77 years was $40.6 \mathrm{pg} / \mathrm{ml}$ and the reference range (2.5th and 97.5th percentiles) was from 24.6 to $136.8 \mathrm{pg} / \mathrm{ml}$ (Table III). For the patients with XLH, as shown in Table IV, the serum FGF23 levels were below the reference range in 4 of 11 subjects, within the range in 4 subjects, and mildly elevated in 3 subjects. The serum FGF23 levels exhibited a wide variation in the patients with XLH, and no significant differences were found when compared with those of the normal controls ( $\mathrm{p}>0.05)$.

\section{Discussion}

Based on the PHEX mutation database (http://www.PHEXdb. mcgill.ca), the frequencies of different mutation types were $25 \%$ frameshifts, $23 \%$ alternative splicing, 22\% missense, $18 \%$ nonsense, $8 \%$ deletion and $4 \%$ polymorphisms. The mutations were scattered throughout the gene and the majority would potentially influence the pattern of post-translational modification of the protein and alter its secondary structure, resulting in the loss of $P H E X$ function $(25,26)$. In this study, we identified 17 different $P H E X$ mutations from 18 unrelated Chinese families, and to the best of our knowledge, 7 were novel.

Three novel missense mutations, namely, Cys77Arg, Gly102Arg and Leu275Pro, were detected. The residue p.77 was amongst the 10 highly conserved cysteine residues that were critical for disulfide-bond formation and protein folding $(25,26)$. Thus, the single base change at this position would likely alter the secondary structure of the protein and render it out of action. Gly102 has been found to be conserved among NEP, PHEX and $E C E-1$ according to the multiple sequence alignment analysis in molecular research (27). Additionally, the glycine to arginine exchange was likely to increase the local charge of the PHEX gene product as arginine was positively charged, while glycine had an uncharged polar group. Therefore, it is possible that this base alteration plays a role in a different spatial conformation. The novel missense mutation detected in exon 7 involved a 
Family 1

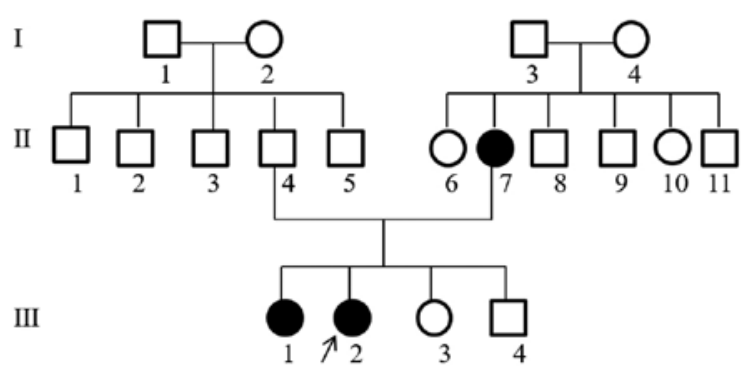

Family 2

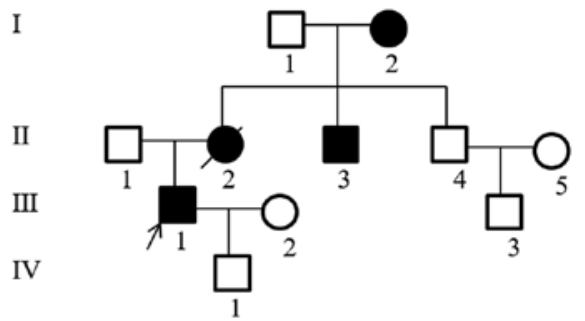

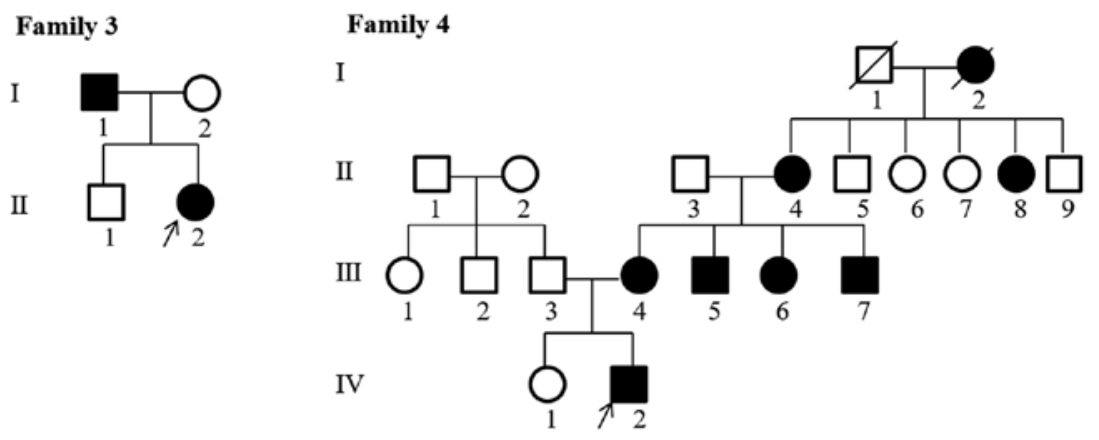

Family 5

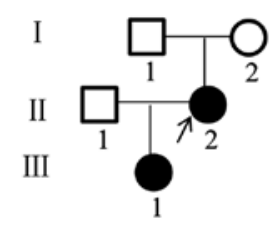

Family 6

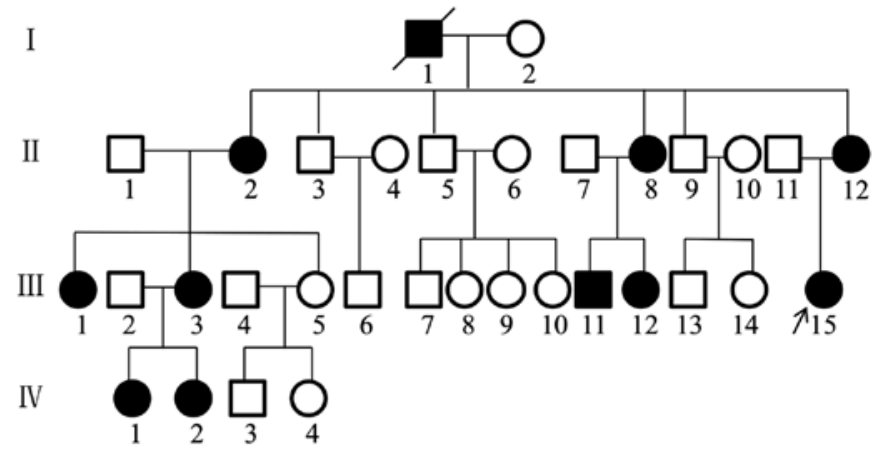

Family 7

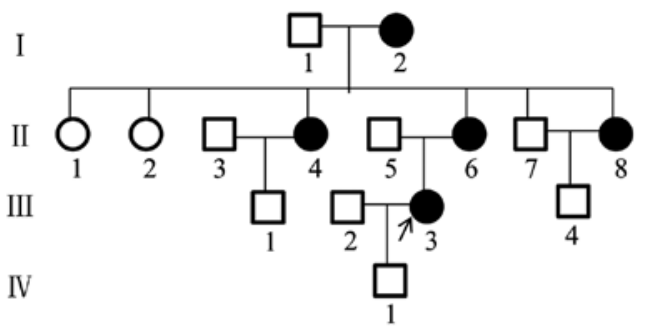

Family 9

I

III

(1)

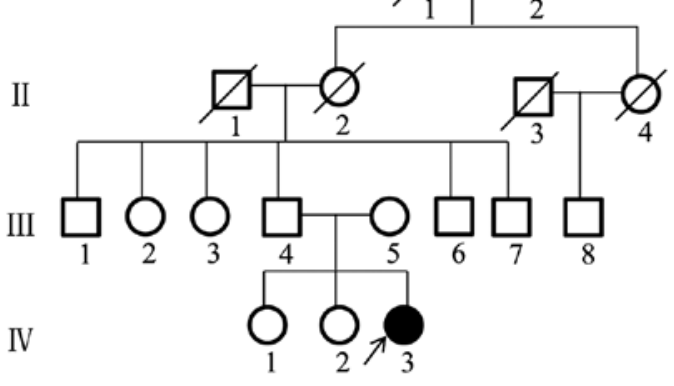

Family 8

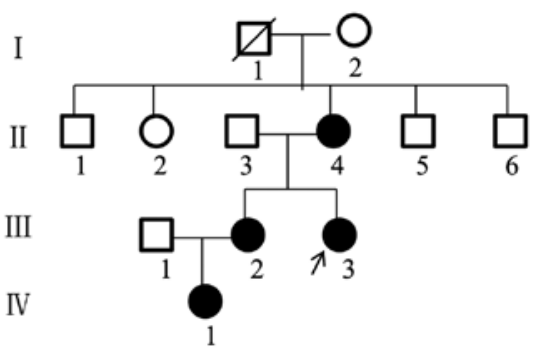

Family 10

Family 11

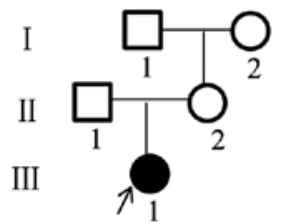

Figure 1. Pedigree of families 1-11 with hypophosphatemic rickets. The black symbols represent the affected individuals, and the open symbols represent the unaffected individuals. The circles and squares indicate females and males, respectively. The arrows identify the probands in the families. 


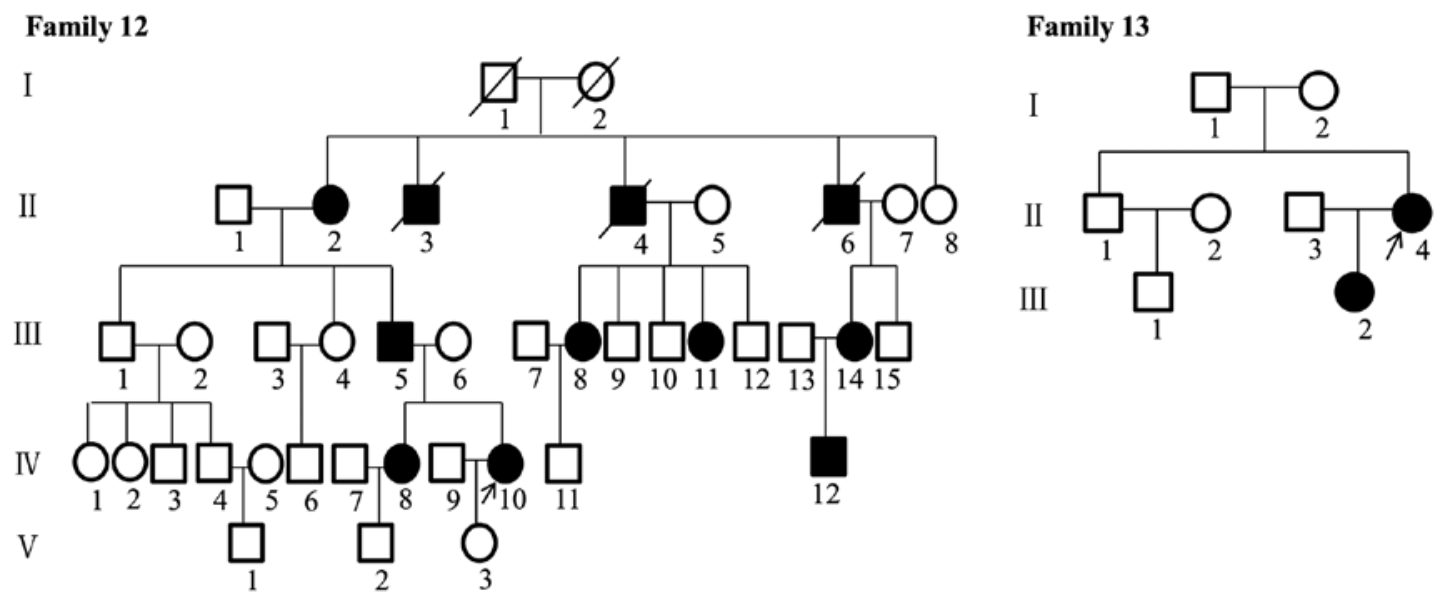

Family 14

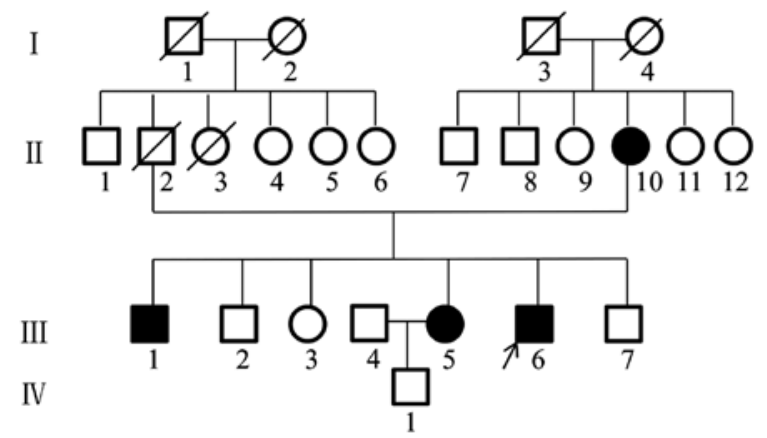

Family 16

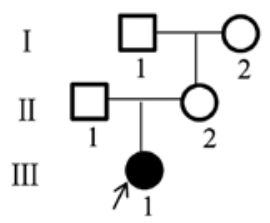

Family 17

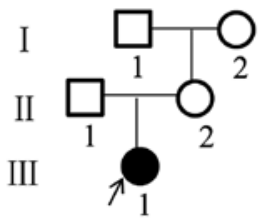

Family 15

I

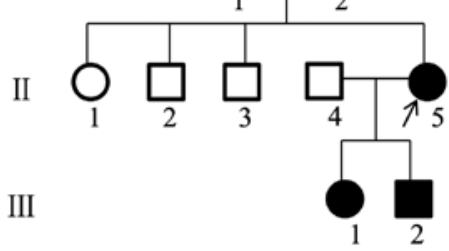

Family 18

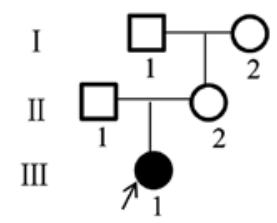

Figure 1. Continued. Pedigree of families 12-18 with hypophosphatemic rickets. The black symbols represent the affected individuals, and the open symbols represent the unaffected individuals. The circles and squares indicate females and males, respectively. The arrows identify the probands in the families.

substitution of proline for leucine at residue 275 . To date, there are only 14 different mutations confirmed in exon 7 and it is among the rarest mutant exons in line with the PHEX mutation database. Moreover, this site was estimated to be conserved with the replaced leucine occurred in both ECE-1 and PHEX. The 3 novel missense mutations identified in this study were shown to be highly conserved under the protein alignment of the PHEX gene from 12 different species and were verified to be pathogenic by bioinformatics tools (PolyPhen-2 and SIFT), which also rendered the evidence of their potential value for the phenotype in patients with XLH.

Three nonsense mutations: Lys432X in exon 11, Arg291X and $\mathrm{G} \ln 311 \mathrm{X}$ in exon 8 were detected in this study, all of which would cause the translation of truncated protein with accidental loss of C-terminal region. It was demonstrated that the C-terminal region in the large extracellular domain was abundant in conserved cysteine residues and contained the zinc-binding motifs in exons 17 and $19(10,11)$. The cysteine residues are responsible for the secondary structure formation and contribute to conformation integrity. The highly conserved zinc-binding motifs among NEP, PHEX, ECE-1 and KELL, are essential for the catalytic activity of the protein. Therefore, these 2 nonsense mutations would inevitably lead to impaired PHEX protein function.

Six of the 17 PHEX mutations were identified as alternative splicing mutations, including 3 splice acceptor mutations and 3 splice donor mutations. Among these, 2 were novel mutations: 436_436+1delAG in the splice donor site of exon 4 and an alteration of $\mathrm{C}$ to $\mathrm{G}$ in intronic sequence 5 ' to initiate exon $11,-1 \mathrm{bp}$ upstream. These 2 splicing mutations were predicted to result in the skipping of exons 4 and 11, respectively. Notably, both of the 2 affected exons comprised one conserved cyteine residue that was relevant for secondary structure transformation and thus protein function. That is, the newly identified $2 \approx$ splicing mutations were associated with the onset of XLH.

The remaining 3 mutations were 1234 delA in exon 11 , c.528delT in exon 5 and 1843dupA in exon 18, characterized as frameshift mutations. The mutation occurring in exon 18 was first reported by Gauche et al (28). It caused the replacement of aspartic acid for threonine at residue 615 and opened a new reading frame for 6 amino acids and deemed to disrupt the overall integrity of the PHEX protein. Moreover, the hetero- 


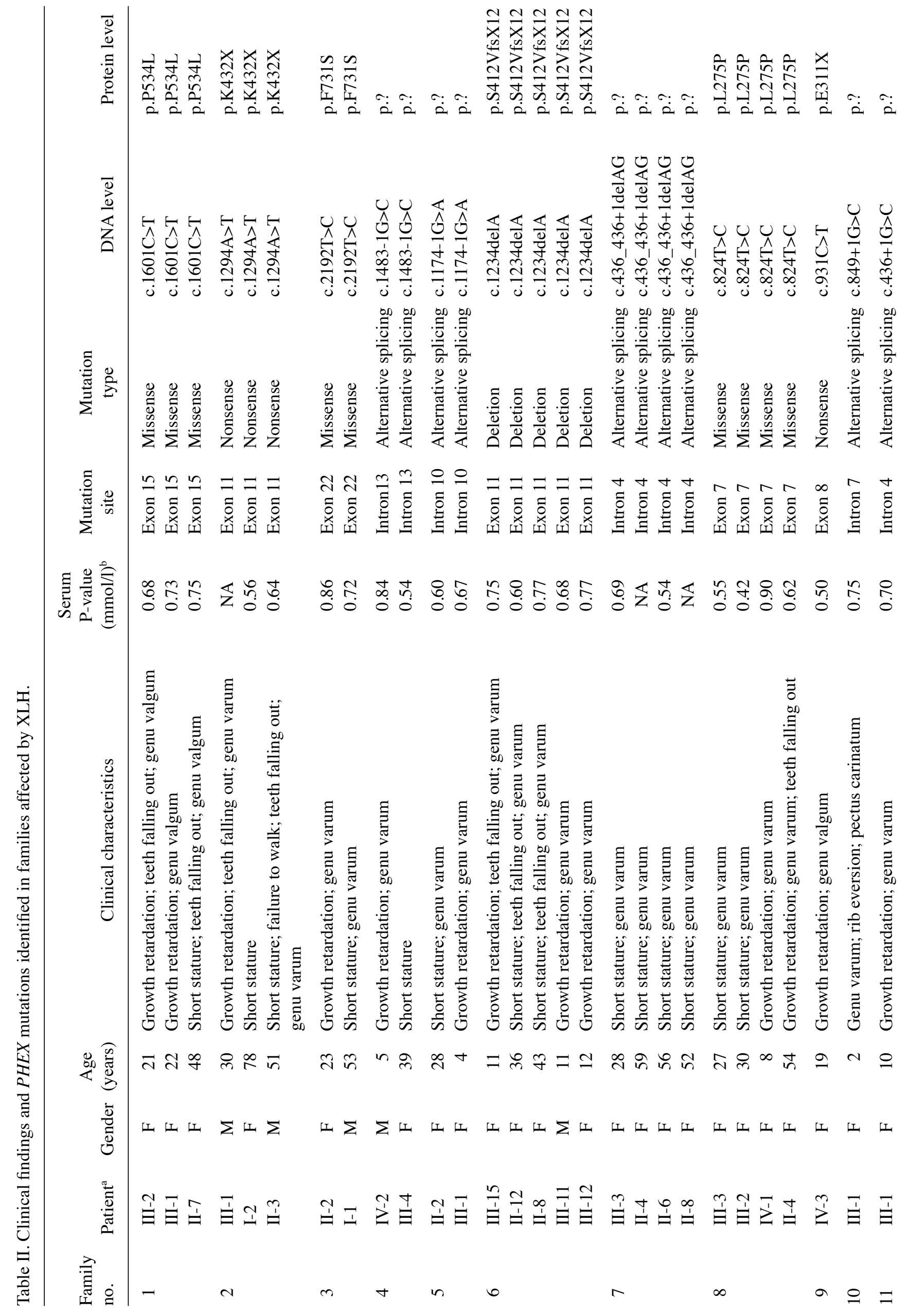




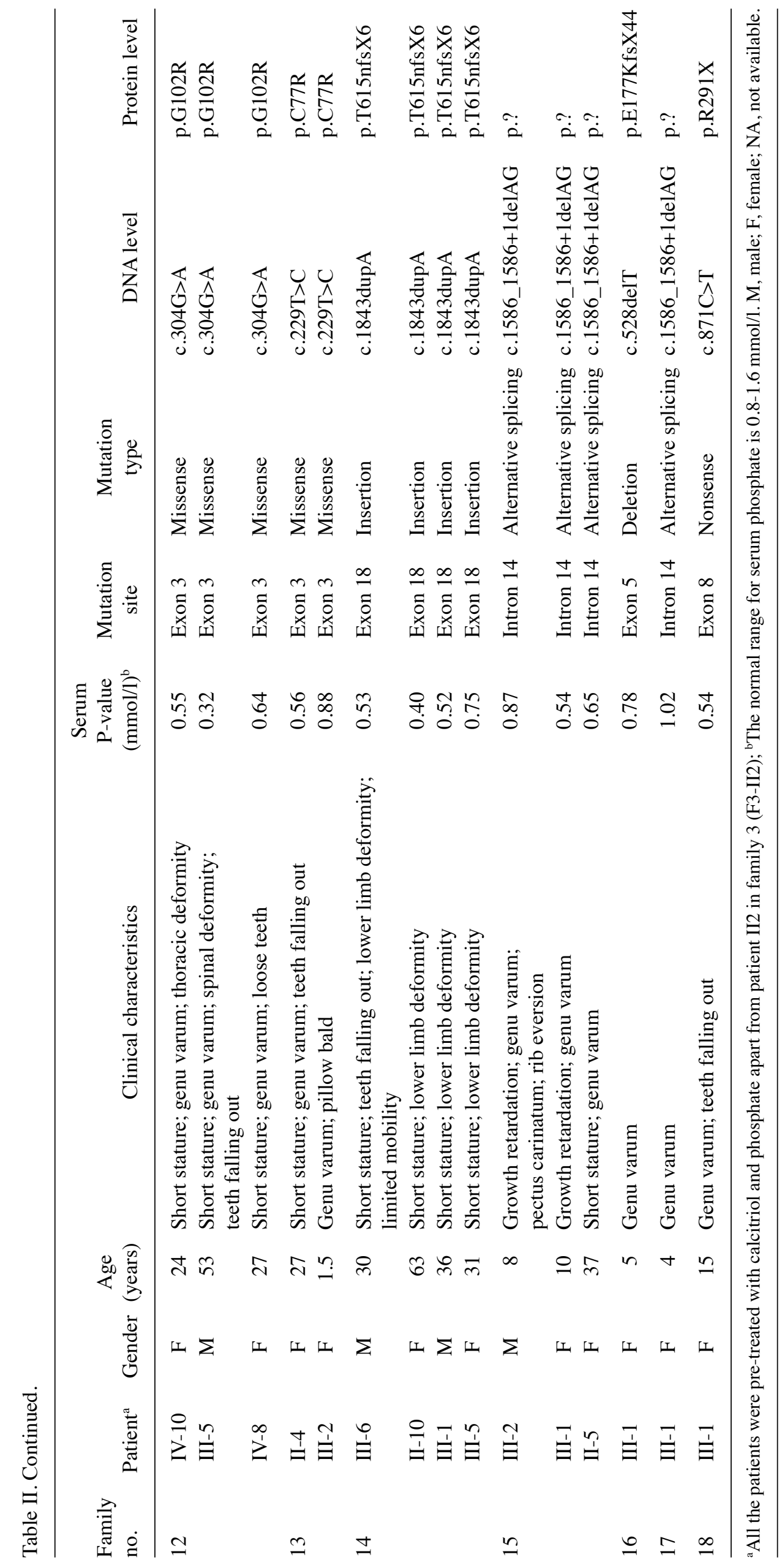




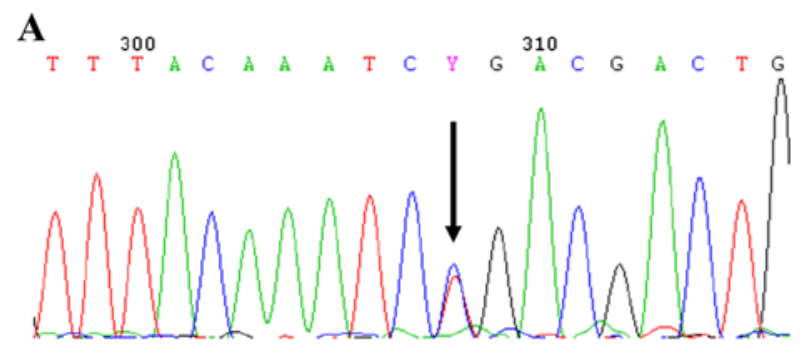

C

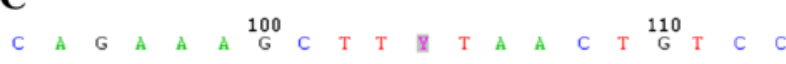

B

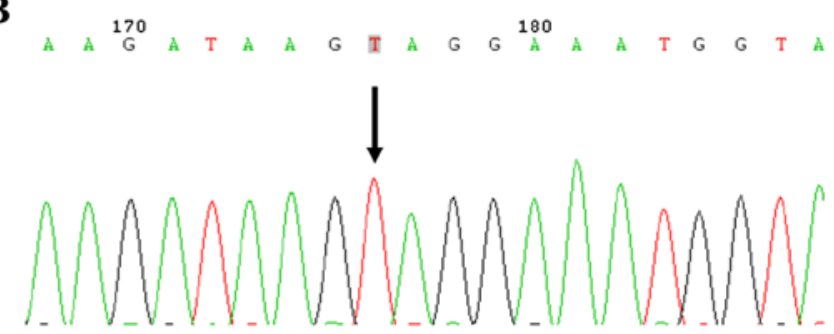

D ${ }_{G}^{100}$ T

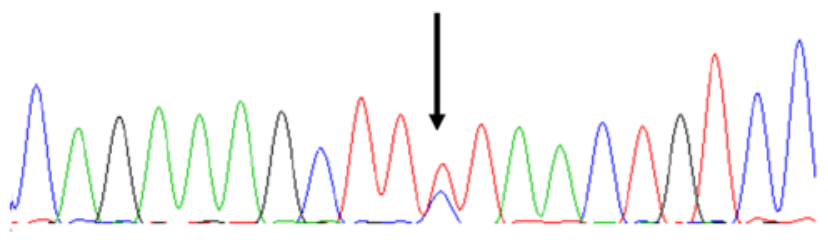

E C A A A T T T A T C T C C C C A C A

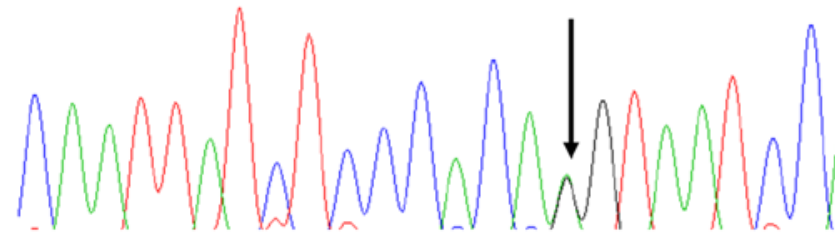

G

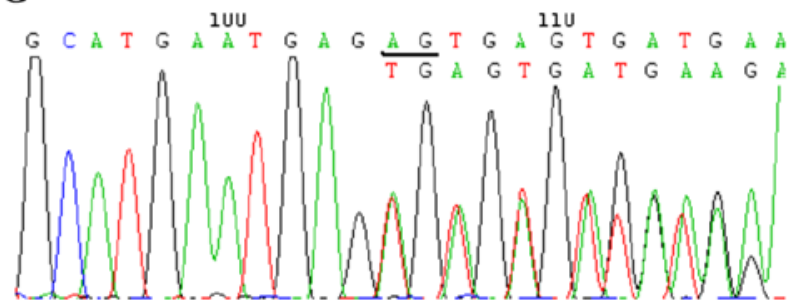

I

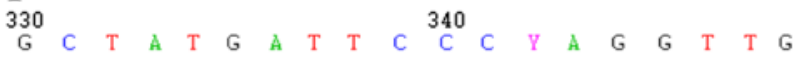

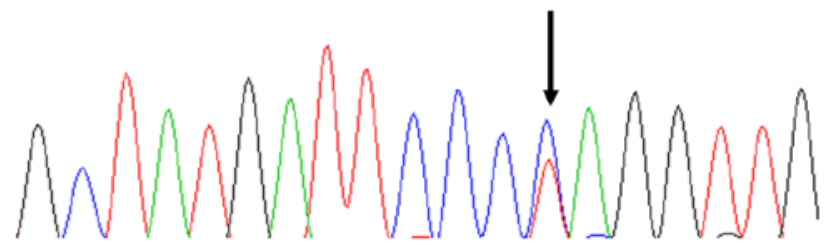

K
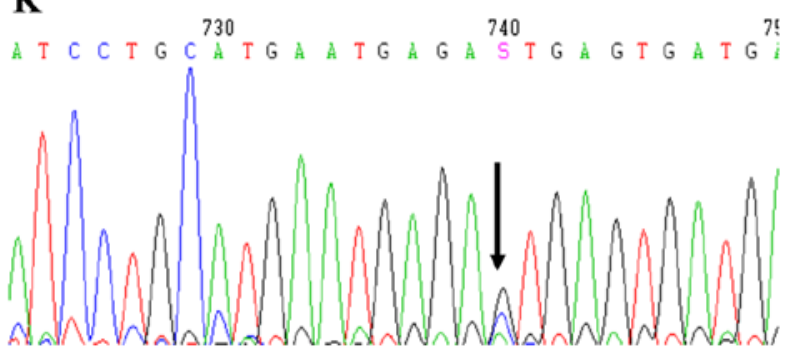

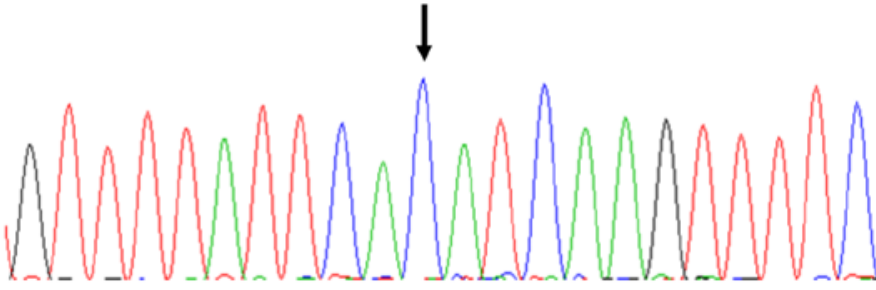

F

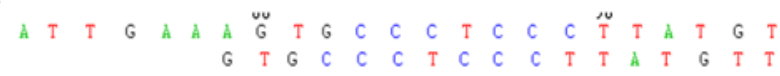

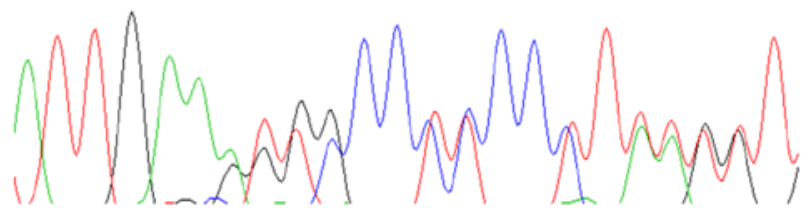

H

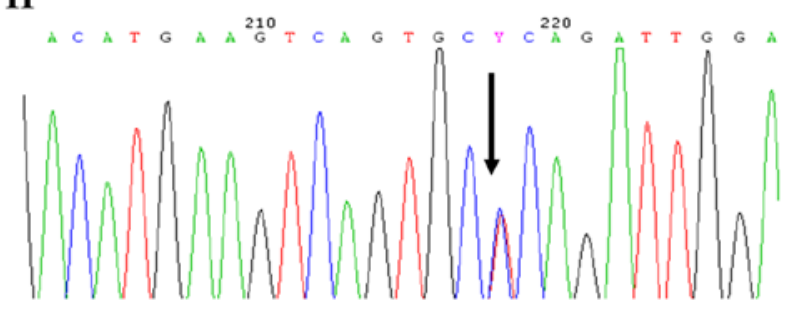

J

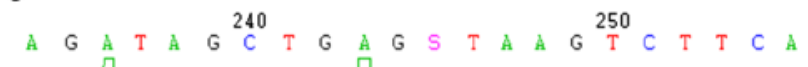

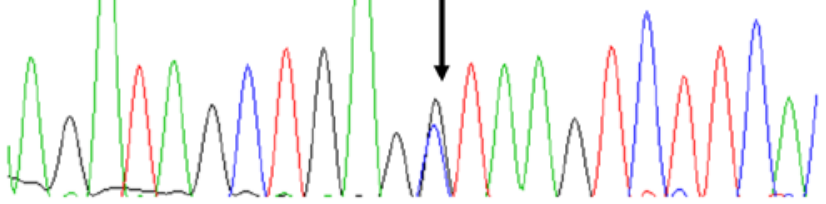

L T G T C T G T G G A T C C T Y G T G A T A A T T T T C T T C C G

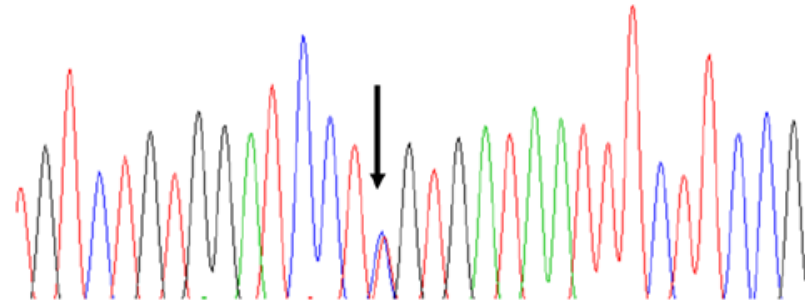

Figure 2. Mutational analyses of phosphate-regulating gene with homology to endopeptidase on the $\mathrm{X}$ chromosome (PHEX) gene in patients with hypophosphatemic rickets. (A) c.1601C $>\mathrm{T}$ in exon 15, (B) c.1294A $>\mathrm{T}$ in exon 11, (C) c.2192T $>\mathrm{C}$ in exon 22, (D) c.1483-1G $>\mathrm{C}$ in intron 13, (E) c.1174-1G $>\mathrm{A}$ in intron 11, (F) c.1234delA in exon 11, (G) c.436_436+1delAG in intron 4, (H) c.824T>C in exon 7, (I) c.931C >T in exon 8, (J) c.849+1G>C in intron 7, (K) c.436+1G>C in intron $4,(\mathrm{~L}) \mathrm{c} .229 \mathrm{~T}>\mathrm{C}$ in exon 3. 

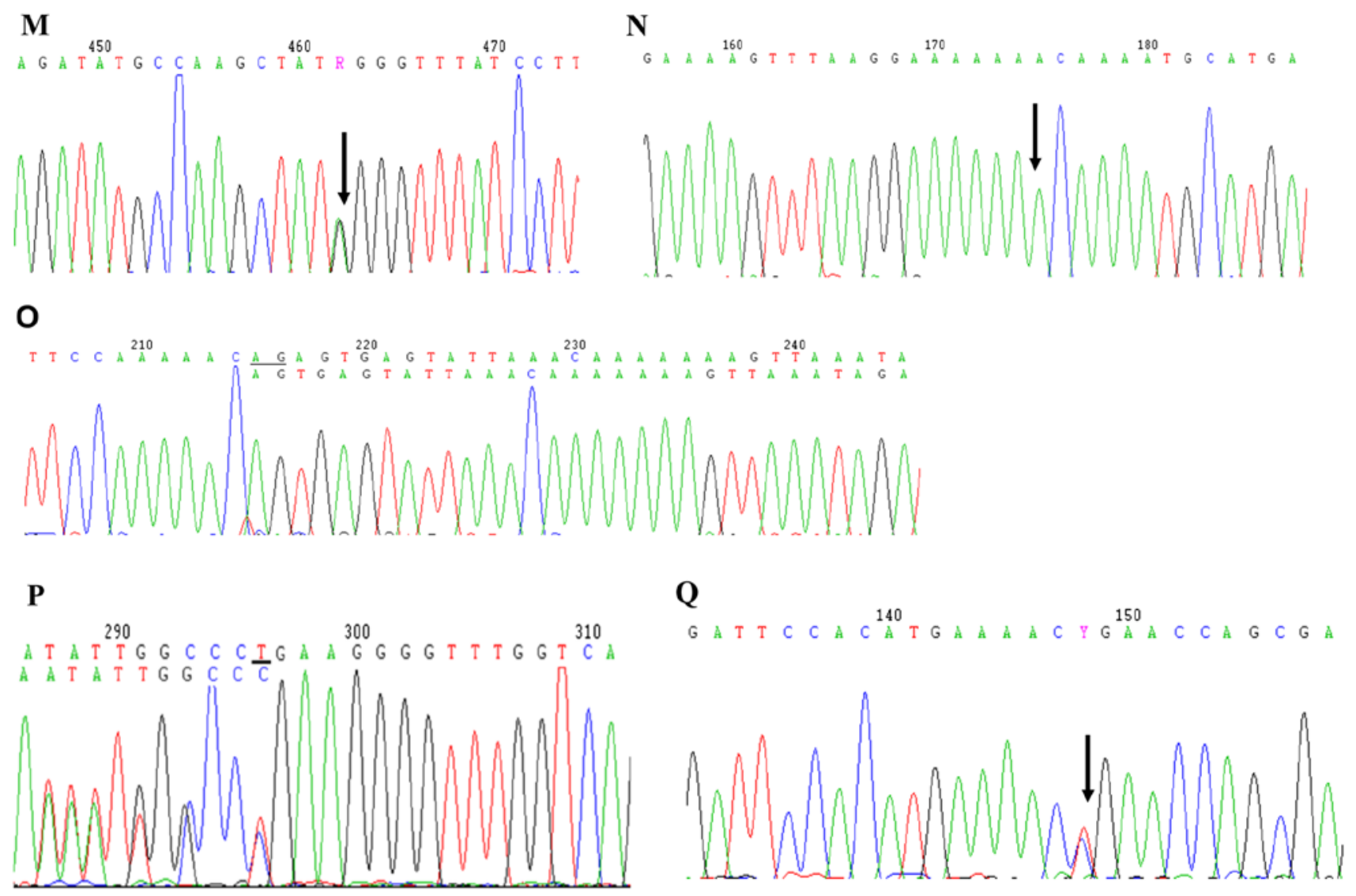

Figure 2. Continued. Mutational analyses of PHEX gene in patients with hypophosphatemic rickets. (M) c.304G>A in exon 3, (N) c.1843dupA in exon 18, (O) c.1586_1586+1delAG in intron 14, (P) c.528delT in exon 5 and (Q) c.871C $>$ T in exon 8.

Species
Homo sapiens
Rattus norvegicus
Danio rerio
Mus musculus
Ailuropoda melanoleuca
Equus caballus
Macaca mulatta
Myotis lucifugus
Oryctolagus cuniculus
Sus scrofa
Anolis carolinensis
Gallus gallus

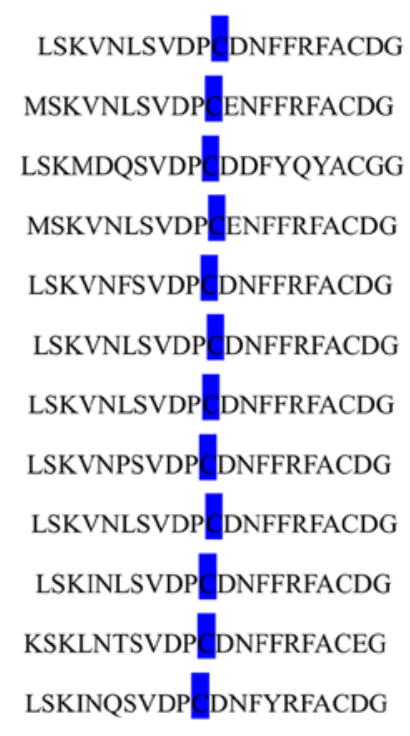

A (p.77)

\begin{tabular}{|c|c|}
\hline Amino acid sequen & \\
\hline NPIPEDMPS & VYPWLRHNVD \\
\hline NPIPEDMPS & VYPWLRHNVD \\
\hline NPIPEDSSSY & IYPWLRQNVD \\
\hline NPIPEDMPS & VYPWLRHNVD \\
\hline NPIPEDMP & YPWLRHNVD \\
\hline NPIPEDMPS & YPWLRHNVD \\
\hline NPIPEDM & YPWLRHNVD \\
\hline NPIPEDMF & PWLRHNVD \\
\hline NPIPEDMPS & YPWLRHNVD \\
\hline NPIPEDMPSY & GIYPWLRRNVD \\
\hline NPIPEDSSSY & GIYPWLRHNVD \\
\hline NPIPEDMSN & GVYPWLRHSVD \\
\hline B & \\
\hline
\end{tabular}

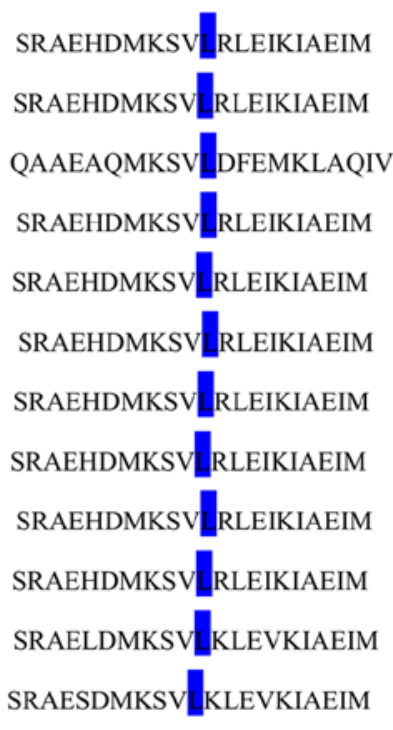

C (p.275)

Figure 3. Evolutionary conservation of the residues p.77, p.102 and p.275. The C77R, G102R and L275P mutations occur at a highly conserved position in phosphate-regulating gene with homology to endopeptidase on the $\mathrm{X}$ chromosome (PHEX) gene, as shown by comparing the corresponding sequences of 12 different species.

zygous deletion of one adenine nucleotide and one thymine nucleotide in exons 11 and 5 were 2 novel mutations identified in our study. These mutations would result in premature termination of the PHEX protein. 
Table III. Serum intact human FGF23 levels in healthy controls.

\begin{tabular}{|c|c|c|c|c|c|c|c|c|c|}
\hline Subjects & $\begin{array}{l}\text { FGF23 } \\
(\mathrm{pg} / \mathrm{ml})\end{array}$ & Subjects & $\begin{array}{l}\text { FGF23 } \\
(\mathrm{pg} / \mathrm{ml})\end{array}$ & Subjects & $\begin{array}{l}\text { FGF23 } \\
(\mathrm{pg} / \mathrm{ml})\end{array}$ & Subjects & $\begin{array}{l}\text { FGF23 } \\
(\mathrm{pg} / \mathrm{ml})\end{array}$ & Subjects & $\begin{array}{l}\text { FGF23 } \\
(\mathrm{pg} / \mathrm{ml})\end{array}$ \\
\hline 1 & 49.3 & 20 & 45.7 & 39 & 26.8 & 58 & 47.2 & 77 & 53.9 \\
\hline 2 & 62.0 & 21 & 20.7 & 40 & 61.0 & 59 & 50.3 & 78 & 56.9 \\
\hline 3 & 37.0 & 22 & 31.9 & 41 & 47.2 & 60 & 29.9 & 79 & 25.8 \\
\hline 4 & 33.0 & 23 & 37.0 & 42 & 60.0 & 61 & 30.4 & 80 & 29.6 \\
\hline 5 & 33.5 & 24 & 34.5 & 43 & 49.3 & 62 & 43.2 & 81 & 40.2 \\
\hline 6 & 26.3 & 25 & 51.3 & 44 & 40.6 & 63 & 40.1 & 82 & 34.0 \\
\hline 7 & 37.0 & 26 & 34.0 & 45 & 47.7 & 64 & 60.5 & 83 & 37.8 \\
\hline 8 & 41.6 & 27 & 93.1 & 46 & 40.1 & 65 & 37.6 & 84 & 41.4 \\
\hline 9 & 153.8 & 28 & 42.1 & 47 & 56.9 & 66 & 53.9 & 85 & 34.1 \\
\hline 10 & 31.9 & 29 & 31.4 & 48 & 35.0 & 67 & 51.3 & 86 & 38.3 \\
\hline 11 & 23.8 & 30 & 68.1 & 49 & 59.0 & 68 & 50.3 & 87 & 47.6 \\
\hline 12 & 44.2 & 31 & 71.7 & 50 & 28.4 & 69 & 30.9 & 88 & 49.0 \\
\hline 13 & 35.0 & 32 & 75.3 & 51 & 136.0 & 70 & 48.3 & 89 & 39.3 \\
\hline 14 & 44.7 & 33 & 65.6 & 52 & 26.3 & 71 & 41.1 & 90 & 47.5 \\
\hline 15 & 116.6 & 34 & 50.3 & 53 & 38.1 & 72 & 64.6 & 91 & 137.4 \\
\hline 16 & 116.1 & 35 & 39.1 & 54 & 35.5 & 73 & 35.0 & 92 & 28.7 \\
\hline 17 & 51.8 & 36 & 39.6 & 55 & 27.4 & 74 & 41.1 & 93 & 35.1 \\
\hline 18 & 33.5 & 37 & 82.4 & 56 & 67.6 & 75 & 30.9 & 94 & 39.6 \\
\hline 19 & 30.9 & 38 & 37.6 & 57 & 33.5 & 76 & 38.1 & 95 & 54.6 \\
\hline
\end{tabular}

FGF23, fibroblast growth factor 23 .

Table IV. Serum intact human FGF23 in patients with XLH.

\begin{tabular}{|c|c|c|c|c|c|}
\hline $\begin{array}{l}\text { Family no.- } \\
\text { Patient }^{\mathrm{a}}\end{array}$ & Gender & Age (years) & $\begin{array}{l}\text { Serum P-value } \\
\qquad(\mathrm{mmol} / \mathrm{l})^{\mathrm{b}}\end{array}$ & $\begin{array}{l}\text { Serum FGF23 } \\
(\mathrm{pg} / \mathrm{ml})^{\mathrm{c}}\end{array}$ & $\begin{array}{l}\text { Mutations in } \\
P H E X \text { gene }\end{array}$ \\
\hline F2-III1 & M & 30 & NA & 38.7 & c.1294A>T (Exon 11) \\
\hline F2-II3 & M & 51 & 0.64 & 40.4 & c. $1294 \mathrm{~A}>\mathrm{T}($ Exon 11$)$ \\
\hline F3-II2 & $\mathrm{F}$ & 23 & 0.86 & 21.1 & c.2192T>C (Exon 22$)$ \\
\hline F5-II2 & $\mathrm{F}$ & 28 & 0.60 & 7.8 & c. $1174-1 \mathrm{G}>\mathrm{A}($ Intron 10$)$ \\
\hline F6-III15 & $\mathrm{F}$ & 11 & 0.75 & 171.5 & c.1234delA (Exon 11) \\
\hline F9-IV3 & $\mathrm{F}$ & 19 & 0.50 & 29.7 & c.931C>T $($ Exon 8$)$ \\
\hline F10-III1 & $\mathrm{F}$ & 2 & 0.75 & 111.4 & c. $849+1 G>C($ Intron 7$)$ \\
\hline F11-III1 & $\mathrm{F}$ & 10 & 0.70 & 15.7 & c. $436+1 \mathrm{G}>\mathrm{C}($ Intron 4$)$ \\
\hline F12-III5 & M & 53 & 0.32 & 24.0 & c.304G>A (Exon 3) \\
\hline F12-IV 10 & $\mathrm{~F}$ & 24 & 0.55 & 162.2 & c. $304 \mathrm{G}>\mathrm{A}($ Exon 3$)$ \\
\hline F12-IV8 & $\mathrm{F}$ & 27 & 0.64 & 143.2 & c.304G>A (Exon 3) \\
\hline
\end{tabular}

${ }^{a}$ All the patients were pre-treated with calcitriol and phosphate apart from patient II-2 from family 3 (F3-II2). ${ }^{\mathrm{b}}$ The normal range for phosphate is 0.8-1.6 mmol/l. ' ${ }^{\mathrm{S}}$ Serum FGF23 in healthy controls ( $\left.\mathrm{n}=95\right)$ : median $40.6 \mathrm{pg} / \mathrm{ml}$, reference range (2.5th and 97.5 th percentiles) $24.6-136.8 \mathrm{pg} / \mathrm{ml}$. FGF23, fibroblast growth factor 23; M, male; F, female.

No hotspot mutations were found in XLH according to the present study in accordance with previous studies on PHEX mutations in Chinese patients (15-21). However, it was valued that exceeding $50 \%$ mutations occurred around exons 18 to 22 in the C-terminal region based on the mutation analyses in PHEXdb and it was speculated that this region may be the critical domain for PHEX function (29). We failed to determine the gene dosage effect on disease severity by comparing the 
phenotypes of hemizygous males to those of heterozygous females from the same family. An evidence-based study also indicated that there was no difference in severity of the disease between genders in mutant Hyp mouse (30). Theoretically, heterozygous females should have a less severe phenotype due to random $\mathrm{X}$-inactivation, the process of transcriptional silencing of one of the $\mathrm{X}$ chromosomes bringing about half of the normal alleles, while males have none. Sabbagh et al owed the absence of gene dose effect to a threshold of PHEX activity that was required for maintaining normal protein function (25). In addition to the gender impact, questions remain as to the possibility of correlations among either mutation location or type with phenotype severity. In the present study, the association between genotype and phenotype in patients with XLH was not observed. It has been proposed that there is a trend towards a more severe phenotype with mutations located in the C-terminal region or with truncating mutations (31); however, this has yet to be verified in a larger sample size.

The aberrant activity of FGF23 was revealed as a common fundamental mechanism for the development of defects in phosphate and vitamin D metabolism in several hypophosphatemic diseases, including ADHR, XLH and tumor-induced osteomalacia (TIO) $(5,32,33)$. The Hyp mouse, an animal model of XLH, provided evidence of increased levels of FGF23 transcripts due to the inactivated mutations of PHEX. Furthermore, an injection of FGF23 antibodies or the deletion of Fgf 23 from the Hyp mouse has been shown to ameliorate or reverse the phosphate metabolic disorders $(14,34)$. These findings strongly indicate the essential role of FGF23 in the regulation of systemic phosphate homeostasis in the Hyp mouse. However, we failed to observe a significant increase in serum FGF23 levels in all affected individuals compared with the healthy controls. Moreover, 4 of 11 patients with XLH even exhibited serum FGF23 levels below the reference range. Despite of the effect of confounding factors, the wide variation in serum FGF23 levels in XLH, as well as the overlapping FGF23 levels in XLH and healthy controls revealed its limited diagnostic value in patients with suspected XLH. Moreover, it was speculated that other factors, such as parathyroid hormone and 1,25-dihydroxyvitamin-D, were responsible for hypophosphatemia in patients with XLH with inappropriate low to normal serum FGF23 levels, and for the normal serum phosphorus in healthy subjects with high serum FGF23 levels $(33,34)$. Additionally, it was suggested that hypophosphatemia alone was not entirely responsible for the skeletal phenotype in XLH and the potential direct local effects of FGF23 may be involved in bone mineralization independent of its systematic action in phosphate homeostasis $(35,36)$.

In conclusion, we identified 17 different mutations in the PHEX gene in 18 unrelated Chinese families with hypophosphatemic rickets and 7 of these were novel. It should be noted that that 6 of the 17 PHEX mutations have been proven to be de novo, which suggests the frequent occurrence of sporadic cases of XLH in the Chinese population. The findings of the present study highlight the major role of PHEX gene mutations in hypophosphatemic rickets and emphasize the significance of genetic diagnosis in suspected cases to ascertain the clinical diagnosis of XLH, enabling timely intervention. Further studies are warranted in order to perform more extensive mutation analyses in affected individuals in order to elaborate the key domain in PHEX and the identification of unequivocal FGF23 function in the pathogenesis of XLH, subsequently, exploring for more effective treatments, not only for XLH, but also for associated diseases sharing similar molecular mechanism.

\section{Acknowledgements}

This study was supported by grants from the National Natural Science Foundation of China (nos. 81370978 and 81170803 to Z.-L.Z., and no. 81200646 to J.-M.G.), the National Basic Research Program of China (no. 2014CB942903), the Shanghai Leading Talents Award (051) to Z.-L.Z., the Science and Technology Commission of Shanghai Municipality (no. 14JC140500) to Z.-L.Z., the Shanghai Municipal Commission of Health and Family Planning (no. 2014ZYJB0009), the Science and Technology Commission of Chongqing Municipality (no. CSTC2013jcyjC00009) to Z.-L.Z.

\section{References}

1. Albright F, Butler A and Bloomberg E: Rickets resistant to vitamin D therapy. Am J Dis Child 54: 529-547, 1937.

2. Beck-Nielsen SS, Brock-Jacobsen B, Gram J, Brixen K and Jensen TK: Incidence and prevalence of nutritional and hereditary rickets in southern Denmark. Eur J Endocrinol 160: 491-497, 2009.

3. Carpenter TO: New perspectives on the biology and treatment of X-linked hypophosphatemic rickets. Pediatr Clin North Am 44: 443-466, 1997.

4. Francis F, Hennig S, Korn B, Reinhardt R, De Jong P, Poustka A, Lehrach H, Rowe PSN, Goulding JN, Summerfield T, et al; The HYP Consortium: A gene (PEX) with homologies to endopeptidases is mutated in patients with X-linked hypophosphatemic rickets. Nat Genet 11: 130-136, 1995

5. White KE, Evans WE, O'Riordan JLH, Speer MC, Econs MJ, Lorenz-Depiereux B, Grabowski M, Meitinger T and Strom TM; ADHR Consortium: Autosomal dominant hypophosphataemic rickets is associated with mutations in FGF23. Nat Genet 26: 345-348, 2000

6. Feng JQ, Ward LM, Liu S, Lu Y, Xie Y, Yuan B, Yu X, Rauch F, Davis SI, Zhang S, et al: Loss of DMP1 causes rickets and osteomalacia and identifies a role for osteocytes in mineral metabolism. Nat Genet 38: 1310-1315, 2006.

7. Li H, Xie H, Liu W, Hu R, Huang B, Tan YF, Xu K, Sheng ZF, Zhou HD, Wu XP and Luo XH: A novel microRNA targeting HDAC5 regulates osteoblast differentiation in mice and contributes to primary osteoporosis in humans. J Clin Invest 119: 3666-3677, 2009.

8. Hu R, Liu W, Li H, Yang L, Chen C, Xia ZY, Guo LJ, Xie H, Zhou HD, Wu XP and Luo XH: A Runx2/miR-3960/miR-2861 regulatory feedback loop during mouse osteoblast differentiation. J Biol Chem 286: 12328-12339, 2011.

9. Li CJ, Cheng P, Liang MK, Chen YS, Lu Q, Wang JY, Xia ZY, Zhou HD, Cao X, Xie H, et al: MicroRNA-188 regulates agerelated switch between osteoblast and adipocyte differentiation. J Clin Invest 125: 1509-1522, 2015.

10. Du L, Desbarats M, Viel J, Glorieux FH, Cawthorn C and Ecarot B: cDNA cloning of the murine Pex gene implicated in $\mathrm{X}$-linked hypophosphatemia and evidence for expression in bone. Genomics 36: 22-28, 1996.

11. Francis F, Strom TM, Hennig S, Böddrich A, Lorenz B, Brandau O, Mohnike KL, Cagnoli M, Steffens C, Klages S, et al: Genomic organization of the human PEX gene mutated in X-linked dominant hypophosphatemic rickets. Genome Res 7: 573-585, 1997.

12. Ruchon AF, Tenenhouse HS, Marcinkiewicz M, Siegfried G, Aubin JE, DesGroseillers L, Crine P and Boileau G: Developmental expression and tissue distribution of Phex protein: Effect of the Hyp mutation and relationship to bone markers. J Bone Miner Res 15: 1440-1450, 2000.

13. Nesbitt T, Fujiwara I, Thomas R, Xiao ZS, Quarles LD and Drezner MK: Coordinated maturational regulation of PHEX and renal phosphate transport inhibitory activity: Evidence for the pathophysiological role of PHEX in X-linked hypophosphatemia. J Bone Miner Res 14: 2027-2035, 1999. 
14. Liu S, Zhou J, Tang W, Jiang X, Rowe DW and Quarles LD Pathogenic role of Fgf 23 in Hyp mice. Am J Physiol Endocrinol Metab 291: E38-E49, 2006.

15. Jap TS, Chiu CY, Niu DM and Levine MA: Three novel mutations in the PHEX gene in Chinese subjects with hypophosphatemic rickets extends genotypic variability. Calcif Tissue Int 88: 370-377, 2011.

16. Xia W, Meng X, Jiang Y, Li M, Xing X, Pang L, Wang O, Pei Y, Yu LY, Sun Y, et al: Three novel mutations of the PHEX gene in three Chinese families with X-linked dominant hypophosphatemic rickets. Calcif Tissue Int 81: 415-420, 2007.

17. Kang QL, Xu J, Zhang Z, He JW, Lu LS, Fu WZ and Zhang ZL: Three novel PHEX gene mutations in four Chinese families with X-linked dominant hypophosphatemic rickets. Biochem Biophys Res Commun 423: 793-798, 2012.

18. Yue H, Yu JB, He JW, Zhang Z, Fu WZ, Zhang H, Wang C, $\mathrm{Hu} \mathrm{WW}, \mathrm{Gu} J \mathrm{M}, \mathrm{Hu} Y \mathrm{YQ}$, et al: Identification of two novel mutations in the PHEX gene in Chinese patients with hypophosphatemic rickets/osteomalacia. PLoS One 9: e97830, 2014.

19. Liu S, Wei M, Xiao J, Wang CY and Qiu ZQ: Three PHEX gene mutations in Chinese subjects with hypophosphatemic rickets and literature review. Zhongguo Dang Dai Er Ke Za Zhi 16: 518-523, 2014 (In Chinese).

20. Yang L, Yang J and Huang X: PHEX gene mutation in a Chinese family with six cases of X-linked hypophosphatemic rickets. J Pediatr Endocrinol Metab 26: 1179-1183, 2013.

21. Yuan L, Wu S, Xu H, Xiao J, Yang Z, Xia H, Liu A, Hu P, Lu A, Chen Y, et al: Identification of a novel PHEX mutation in a Chinese family with X-linked hypophosphatemic rickets using exome sequencing. Biol Chem 396: 27-33, 2015.

22. Adzhubei IA, Schmidt S, Peshkin L, Ramensky VE, Gerasimova A, Bork P, Kondrashov AS and Sunyaev SR: A method and server for predicting damaging missense mutations. Nat Methods 7: 248-249, 2010.

23. Ng PC and Henikoff S: SIFT: Predicting amino acid changes that affect protein function. Nucleic Acids Res 31: 3812-3814, 2003.

24. Smith ER, McMahon LP and Holt SG: Method-specific differences in plasma fibroblast growth factor 23 measurement using four commercial ELISAs. Clin Chem Lab Med 51: 1971-1981, 2013.

25. Sabbagh Y, Boileau G, Campos M, Carmona AK and Tenenhouse HS: Structure and function of disease-causing missense mutations in the PHEX gene. J Clin Endocrinol Metab 88: 2213-2222, 2003.

26. Beck-Nielsen SS, Brixen K, Gram J and Brusgaard K: Mutational analysis of PHEX, FGF23, DMP1, SLC34A3 and CLCN5 in patients with hypophosphatemic rickets. J Hum Genet 57: 453-458, 2012.
27. Rowe PS, Oudet CL, Francis F, Sinding C, Pannetier S, Econs MJ, Strom TM, Meitinger T, Garabedian M, David A, et al: Distribution of mutations in the PEX gene in families with X-linked hypophosphataemic rickets (HYP). Hum Mol Genet 6: 539-549, 1997.

28. Gaucher C, Walrant-Debray O, Nguyen TM, Esterle L, Garabédian M and Jehan F: PHEX analysis in 118 pedigrees reveals new genetic clues in hypophosphatemic rickets. Hum Genet 125: 401-411, 2009

29. Filisetti D, Ostermann G, von Bredow M, Strom T, Filler G, Ehrich J, Pannetier S, Garnier JM, Rowe P, Francis F, et al: Non-random distribution of mutations in the PHEX gene, and under-detected missense mutations at non-conserved residues. Eur J Hum Genet 7: 615-619, 1999.

30. Qiu ZQ, Tenenhouse HS and Scriver CR: Parental origin of mutant allele does not explain absence of gene dose in X-linked Hyp mice. Genet Res 62: 39-43, 1993.

31. Holm IA, Nelson AE, Robinson BG,Mason RS, Marsh DJ,Cowell CT and Carpenter TO: Mutational analysis and genotype-phenotype correlation of the PHEX gene in X-linked hypophosphatemic rickets. J Clin Endocrinol Metab 86: 3889-3899, 2001.

32. Jonsson KB, Zahradnik R, Larsson T, White KE, Sugimoto T, Imanishi Y, Yamamoto T, Hampson G, Koshiyama H, Ljunggren $\mathrm{O}$, et al: Fibroblast growth factor 23 in oncogenic osteomalacia and X-linked hypophosphatemia. N Engl J Med 348: 1656-1663, 2003.

33. Weber TJ, Liu S, Indridason OS and Quarles LD: Serum FGF23 levels in normal and disordered phosphorus homeostasis. J Bone Miner Res 18: 1227-1234, 2003.

34. Aono Y, Yamazaki Y, Yasutake J, Kawata T, Hasegawa H, Urakawa I, Fujita T, Wada M, Yamashita T, Fukumoto S and Shimada T: Therapeutic effects of anti-FGF23 antibodies in hypophosphatemic rickets/osteomalacia. J Bone Miner Res 24: 1879-1888, 2009

35. Sapir-Koren R and Livshits G: Bone mineralization is regulated by signaling cross talk between molecular factors of local and systemic origin: The role of fibroblast growth factor 23 . Biofactors 40: 555-568, 2014

36. Sitara D, Kim S, Razzaque MS, Bergwitz C, Taguchi T, Schüler C, Erben RG and Lanske B: Genetic evidence of serum phosphate-independent functions of FGF-23 on bone. PLoS Genet 4: e1000154, 2008. 